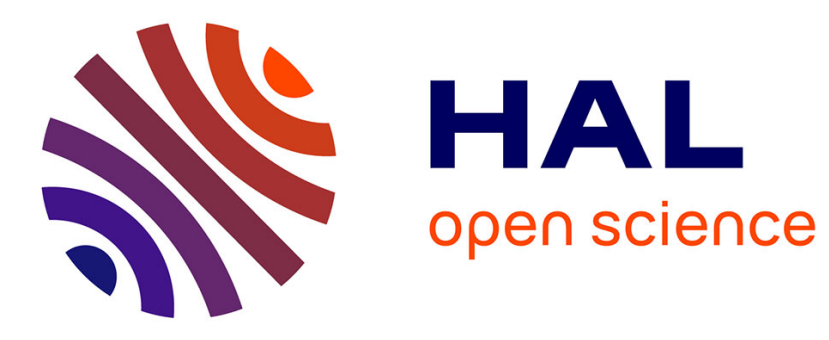

\title{
Understanding normal cardiac development using animated models
}

Jean-Marc Schleich, Jean-Louis Dillenseger, Stéphane Andru, Jean-Louis Coatrieux, Claude Almange

\section{To cite this version:}

Jean-Marc Schleich, Jean-Louis Dillenseger, Stéphane Andru, Jean-Louis Coatrieux, Claude Almange. Understanding normal cardiac development using animated models. IEEE Computer Graphics and Applications, 2002, 22 (1), pp.14-19. 10.1109/38.974513 . inserm-00131250

\section{HAL Id: inserm-00131250 https://www.hal.inserm.fr/inserm-00131250}

Submitted on 15 Feb 2007

HAL is a multi-disciplinary open access archive for the deposit and dissemination of scientific research documents, whether they are published or not. The documents may come from teaching and research institutions in France or abroad, or from public or private research centers.
L'archive ouverte pluridisciplinaire HAL, est destinée au dépôt et à la diffusion de documents scientifiques de niveau recherche, publiés ou non, émanant des établissements d'enseignement et de recherche français ou étrangers, des laboratoires publics ou privés. 


\section{HAL author manuscript}

This material is presented to ensure timely dissemination of scholarly and technical work. Copyright and all rights therein are retained by authors or by other copyright holders. All persons copying this information are expected to adhere to the terms and constraints invoked by each author's copyright. In most cases, these works may not be reposted without the explicit permission of the copyright holder.

\section{Understanding the normal cardiac development using animated models}

Jean-Marc Schleich*, Jean-Louis Dillenseger**, Stéphane Andru, Jean-Louis Coatrieux**, Claude Almange*

*Département de Cardiologie et Maladies Vasculaires, Hôpital de Pontchaillou, CHR Rennes, France.

**Laboratoire de Traitement du Signal et de l’Image, Université de Rennes 1, Rennes, France

\section{Corresponding author}

Jean-Marc Schleich, M.D.

Département de Cardiologie et Maladies Vasculaires, Hôpital de Pontchaillou, 35033 Rennes cedex 09, France. Email: jean-marc.schleich@chu-rennes.fr 


\section{Introduction}

The learning of the heart development is essential in Medical Education and furthermore in Cardiology Education. It allows to understand the morphology of a normal heart and also to explain the formation of congenital heart defects. However, the learning of cardiac embryology is difficult because the processes underlying the heart formation are complex. This complexity is induced by the fact that an organ that is as complicated as the heart, is generated only from a couple of cells.

In a few words, the main steps of the heart development are the following. Around 18 to 20 days of development, originating from the embryonic cells, a tube (the primitive heart tube) with a unique cavity forms the future embryonic heart. This tube then twists itself in order to get the future anatomic topology. The next steps of the formation are the partitioning of the unique cavity in two atria and two ventricles by tissue growing. The development of the valves achieves as last. All these development steps follow one another in a dynamic process.

The heart development is not easy to learn and to understand because not only the spatial arrangement of the anatomical structures but also the dynamic evolution of these structures has to be perceived in it whole complexity.

Classically two main ways can be used to learn the heart development: lectures or textbooks. But none of these teaching modalities is well adequate to describe the spatio-temporal complexity of the heart development. An idea of the difficulty of such a description can be seen on the sidebar dealing about the development of the cardiac outflow tracts. Classically, the descriptions are illustrated by sketches as in figure $1^{1}$ to help mental visualization or representation (hence the understanding) of the spatio-temporal evolution of this phenomenon.

However, such drawings and sketches are not effective enough because two-dimensional pictures only give a partial impression of the geometry of the objects. Moreover, the dynamics of structural evolution can only be rendered by a limited sequence of static images. This inadequacy to represent complex spatial developments is a real and major limitation. Tremendous efforts are then required to graphically illustrate the generation and the temporal transformation of most cardiac structures.

It is well established that advanced computer graphics can provide substantial improvements for teaching in medicine (see for example the special issue "Imaging in Medical Education"²) through the design, the modeling, the visualization and the manipulation of 3-D objects, as well as to the production of animated movies. This paper shows how animated models can significantly help the understanding of the complex changes, which occur during normal heart development. 


\section{Construction of the model}

The overall objective of the project was to design and develop a 3-D model capable to render the dynamics of the various stages of normal heart development, beginning at fertilization. In the biomedical field classically, anatomical modeling is achieved through reconstruction from 3-D medical images ${ }^{3}$. However, the morphological complexity and the extreme difficulties inherent in the acquisition of real images at all the stages of embryonic human heart development led us to design the heart model rather than reconstructing it (other medical applications use the same combination of 3-D shape design and generic model animation ${ }^{4}$ ). In addition, that option enhances the generic aspect of the model, making it more suitable for educational demonstration or teaching.

This work implies a close cooperation between physicians and the graphic modeler in charge of designing the 3D animated sequence. A development framework was used to input medical knowledge or clinical hypotheses into the final 3-D product. This development framework can be summarized as follows:

\section{Medical knowledge}

In a first step, medical knowledge is defined/formulated by a multidisciplinary group of physicians including cardiologists, pediatrician-cardiologists and embryologists. A literature review of the domain and the knowledge of embryology generally lead to sketch shapes for several stages of normal heart development. They are only a few quantitative data available on the model, even in the medical literature. The morphology, the size or the proportion of shapes are mainly deduced from the literature data and in some case hypothesized by the physician pool. In such specific cases as atrial partitioning and because of the scarcity of knowledge in this medical field, several shapes can be hypothesized, from which experts will retain the one they deem most plausible.

\section{3-D Modeling}

The 3-D sequence is designed by a graphic modeler and supervised by a physician. A storyboard is first defined. The overall sequence is organized around about 30 static 3-D shapes, which represent the main stages of heart development. The modeling process then takes place as follows: a) 3-D modeling of the main 3-D shapes and b) production of 3-D animations to link the main shapes. This comprehensive 3-D design process is performed using Newtek's LightWave ${ }^{\mathrm{TM}}$ [5.5] product (http://www.newtek.com/products/lightwave/index.html) running on a Personal Computer (Pentium II Xeon biprocessor 450MHz) with 192 Mo. of RAM and an Evans\&Sutherland AxelGalaxy 3-D graphic card.

a) Experts' representations of the 3-D shapes are transferred to the modeler by means of 2-D sketches and also by some 3-D plaster sketches when the 3-D shapes are too complex to be represented in two dimensions 
(figure 2). The 3-D shapes are hewed by visually driven deformations of a few basic 3-D patterns. The modeler acts directly on the control points of the shape surfaces described by MetaNURBS (the surface model used in the LightWave ${ }^{\mathrm{TM}}$ package). He also assigns the basic colors to the various parts of the structures. At this stage of modeling, only unsophisticated surface attributes are assigned. The result of the modeling is a polygonal model of the shape (figure 3). In order to have an idea about the complexity of the shape, the opened heart presented on figure 3 is composed by more than 16,000 polygons. The shapes so generated are submitted to the medical experts for validation. This continuous interchange between physicians and the graphic modeler occasionally produces feedback into medical knowledge: certain medical hypotheses based on literature data were found to be erroneous when interpreted in three dimensions.

b) Animation can be produced by two different methods: morphing from one shape to another or specific shape deformation over time. The former is the preferred method because globally the changes between development stages are smooth and deformations are relatively minor. LightWave ${ }^{\mathrm{TM}}$ propose a point-to-point linear morphing process. The main shapes beginning and finishing an animation are modeled in parallel so as to achieve a point-to-point correspondence. In case of larger amplitude deformation, intermediate shapes have to be modeled to smooth the morphing (on average, 2 or 3 such intermediate shapes are used; figure 7 is a really good example of a complicated motion, at least 5 intermediate shapes have be modeled to describe the cardiac loop formation). The latter method is based on the creation of a shape skeleton (Bones in LightWave ${ }^{\mathrm{TM}}$ terminology) and animation is obtained through direct action on this skeleton. This method has been used for more complex deformations (e.g., spermatozoid flagellae in figure 4). For the overall dynamic sequence, more than 7,000 3-D objects have been created.

\section{Formal medical validation}

The multidisciplinary group of physicians regularly evaluates the shapes and animations in relation to current knowledge in embryology. The 3-D shapes as well as the validity of medical hypotheses are submitted to critical examination and possible modifications may be fed back to the modeler.

\section{Final modeling}

After completion of the previous 3-D shape model, surface properties must be incorporated (shading attributes, shading interpolation -Gouraud-, transparencies, color and texture). The color attributes of the objects are selected to be as natural as possible or to follow conventional medical iconographic rules. The realistic aspect is also enhanced by 2-D color textures extracted from medical pictures (e.g., photography of a real myocard) and by some 3-D textures (bumps). An example of this process can be seen on figure 9 which is the enhanced 
version of the $3 \mathrm{D}$ object shown on figure 3. After these refinements, the various development stages can be visualized as a time-coded sequence of animated 3-D objets.

\section{Visualization of the development stages}

The main way to display the morphogenetic development stages consists on a movie showing the evolution of the heart formation. LightWave ${ }^{\mathrm{TM}}$ offers the possibility to position cameras in the 3-D scene and to select rendering parameters. In this specific case, there were only a few matte objects. Without losing realism, we chose a rendering without casted shadows and multiple reflections. LightWave ${ }^{\mathrm{TM}}$ stores the rendered images of the sequence in Targa32 format. The image resolution is $728 x 576$ pixels in order to match the picture to the PAL video standard. The final animation is finally edited with Adobe Premiere software and is available in AVI format (two examples can be found on http://virtual-heart-development.univ-rennes1.fr/anglais/generale.htm animations button). But this animation is not self-explaining as it. Oral explanations and identification signs are post-processed on the video in order to describe precisely the anatomy and it mutation and to highlight some particularities of this anatomy or some specific phenomenon. The objective of this animation is really to teach the normal heart development. The final animation is about 12' long containing more than 18,000 frames.

\section{Results}

The overall process of the model design from the medical definition of the sequence to the final modeling and validation took about 3 years. The pictures presented here highlight some important stages of the overall cardiac morphogenesis. Chronologically, the first visible phase is fertilization (figure 4). In this sequence, each spermatozoid has a skeleton on which we act to create motion. The texture of the ovule takes inspiration from real microscopy images.

After a few cellular divisions, 6 or 7 days after fecundation, the human conceptus becomes a morula (mulberry shaped). On the top of the open morula (figure 5), we can see an inner cell mass called the embryoblast and its two layers: the ectoblast (the future embryo) with the amniotic cavity in blue and the entoblast (the future yolk sac) in yellow. The choice of colors follows the rules of major medical textbooks.

On Day 20 (figure 6), the vascular network of the embryo forms a bow which is seen by transparency within the embryo on the left and extracted from the embryo on the right. The rotation around the embryo or the extracted vascular bow offers a better understanding of its spatial topology. In the center of the bow, a bulb will form the future heart. At this stage of development, the heart is just a beating tube.

Figure 7 displays a time sequence for cardiac loop formation, from Day 21 to Day 28. The heart tube folds into an S-shape to form a dextro-ventral (right-front) convexity loop also called D-loop. After this folding, the heart 
takes its final topology (the future ventricles is oriented downwards with the great artery on the top and the future atria backwards and upwards). This transformation is usually relatively complex to describe but can be very easily understood just by viewing the animation.

Figure 8 takes back to the partitioning of the conus (a, the upper part of the ventricular chamber) and the truncus (b, in yellow on the model) described in the sidebar. The model allows to open up the heart and to visualize its internal structures. The various steps of partitioning are now clearly detectable on the pictures, providing a better description than the sketch in figure 1. Moreover, the animation of the ridges growing improves significantly the perception of the complex topology of this partitioning

In figure 9, the ventricular chamber development unfolds by the formation of the valves and cords. On this picture, the inner wall texture was picked from a photography of a real embryo heart. The texture of the valves, aorta (e) and pulmonary artery (f, both at the top of the shape) was taken from an anatomy textbook.

The purpose of this study is now to integrate the animation into a Computer Aided Teaching tool which will include exhaustive explanatory texts, interactive commentaries, a set of bibliographic references and also some annotated fetal echocardiographs. The spatial topology of certain key moments of the morphogenesis is really complex to understand. It is the reason why we would select a set of 3-D objects from the 3-D model animations that could be interactively manipulated by the medical students to ensure an optimal integration of the spatial topology. It is our hope that this tool will help self-learning of normal heart development. This tool is actually available on a CDROM and the animation alone on videotape. More examples of images or animations can be found on: http://virtual-heart-development.univ-rennes1.fr/anglais/generale.htm.

\section{Conclusion}

These illustrations demonstrate that animated models significantly improve the understanding of complex phenomena. This animation (in the form of commented videotape) has already been used for several courses on normal heart development. Professors' assessment (also based on students' remarks and comments) is that this type of animation greatly helps in understanding normal heart development, both spatially and temporally. The other positive aspect is that memorizing is much easier than from classical lessons based on sketches. That type of animation has acquired an irreversible status within the learning process. However, used alone, this animation has a major drawback. The students mainly focus their attention on the animation, i.e., on the spatio-temporal aspects of the overall development process. Precise anatomy details (names and functions) are usually not simultaneously integrated during visualization of the sequence and must be learnt afterwards. Nevertheless, we believe that the use of 3D animated models combined with explanatory texts, interactive commentaries, a set of 
bibliographic references and interactively manipulable 3D objects can play a major role in self-learning of normal heart development

\section{Acknowledgments}

The animation presented in this paper was awarded first Prize at the $7^{\text {th }}$ International Medical Film and Multimedia Festival, FILMED2000, Amiens, France, in the category "Cardiology, Cardiovascular diseases and Cardiovascular surgery”.

The authors wish to thanks Dr Lucille Houyel, Pr Antoine Casasoprana and Pr François Picard for their help in the validation of the model. They wish also to thank Stéphane Andru who designed the 3-D objects and the animated sequence. English proofreading by Philip Rousseau-Cunningham.

\section{References}

1. W. J. Larsen, Human Embryology, 1rst edition, Churchill Livingston Inc., New York, 1993.

2. P. Dev, "Imaging in Medical Education", IEEE Computer Graphics \& Applications, Special Issue on Imaging in Medical Education, 19, 3, 1999.

3. J. Kaye, D. N. Metaxas, F. P. Primiano Jr., "A 3D Virtual Environment for Modeling Mechanical Cardiopulmonary Interactions", Proc. CVRMed-MRCAS'97, Lectures Notes in Computer Science 1205, Springer Verlag, 1997, pp. 389-398.

4. T. Berlage, "Augmented-Reality Communication for Diagnostics Tasks in Cardiology", IEEE Trans. on Information Technology in Biomedicine, 2, 3, 1998, pp. 169-173.

\section{Captions}

Figure 1: Sketches illustrating the development of the cardiac outflow tracts (from "Human Embryology”, W. J. Larsen, Churchill Livingston Inc).

Figure 2: An example of a 2-D sketch and a 3-D plaster model used to transfer the medical anatomical knowledge to the modeler. The 3-D physical model corresponds to the third and fourth image of figure 8.

Figure 3: Heart model under construction: wire frame and filled polygons. The refined model with texture can be seen on the fourth image of figure 8 .

Figure 4: Fecundation (fertilization) of the ovule. 
Figure 5: Day 6 and 7 after fecundation: the opened morula (mulberry shaped) with the embryoblast on the top which is formed by (in blue) the ectoblast (the future embryo) with the amniotic cavity and (in yellow) the entoblast (the future yolk sac).

Figure 6: Day 20. The primitive heart tube and the aorta can be seen within the embryo by transparency on the left side and isolated on the right side.

Figure 7: From Day 21 to Day 28. The formation of the cardiac loop where the heart tube is folded into a Sshape. The heart takes its final topology (the future ventricles is oriented downwards with the great artery on the top and the future atria backwards and upwards).

Figure 8: The formation of the cardiac outflow tracts: the partitioning of the conus (a) and the truncus (b), in yellow on the pictures. The dextrodorsal (back right) and sinistroventral (front left) conus ridges, isolated on the first picture, partition the conus by a helical outgrowth, into two cavities: the subpulmonary (c) and the subaortic (d) coni. The truncus is partitioned from the bottom upward from aortico-pulmonary swellings leading to the formation of the aorta (e) and the pulmonary artery (behind the aorta).

Figure 9: The mitral (a) and tricuspid (c) valves separating respectively the left and the right atria from the corresponding ventricle. This model of the opened heart shows the mitral valve (a) and the left ventricle (b), the tricuspid valve (c) and the right ventricle (d), the aorta (e) and the pulmonary artery (f).

\section{Sidebar}

An example of the classic way used to describe a part of the normal heart development: the cardiac outflow $\underline{\text { tracts. }}$

The presentation of the development of the cardiac outflow tracts is a good example of the difficulties to describe spatio-temporal phenomenon. From Day 26, two embryological areas of the ventricular outflow tract, the conus and the truncus, partition in order to form the pulmonary infundibulum (the right ventricle ejection chamber), the aorta and the pulmonary artery. The sequence of the partitioning is the following (figure 1$)^{1}$. Picture A: dextrodorsal (back right) and sinistroventral (front left) ridges (called truncoconal septae on the picture) appear along the ventricular outflow tract. These two opposite ridges grow into a helix (picture B) and join together so as to separate the conus and truncus in two parts (picture C). Partitioning unfolds from bottom to top in a helix because the ridges grow spatially in a rotational shift. Picture D: the conus is divided into two conal cavities (the dextroventral one -at the beginning of the blue arrow- is called pulmonary infundibulum and 
the sinistrodorsal one will be absorbed after the partitioning). The truncus divides into the aorta (red arrow) and pulmonary artery (blue arrow). The illustration gives only a partial impression of the overall dynamic process. Animation of 3-D models (illustrated on figure 8 by some frames picked out of the animation sequence) improves significantly the perception of the form's evolution. 
Figures
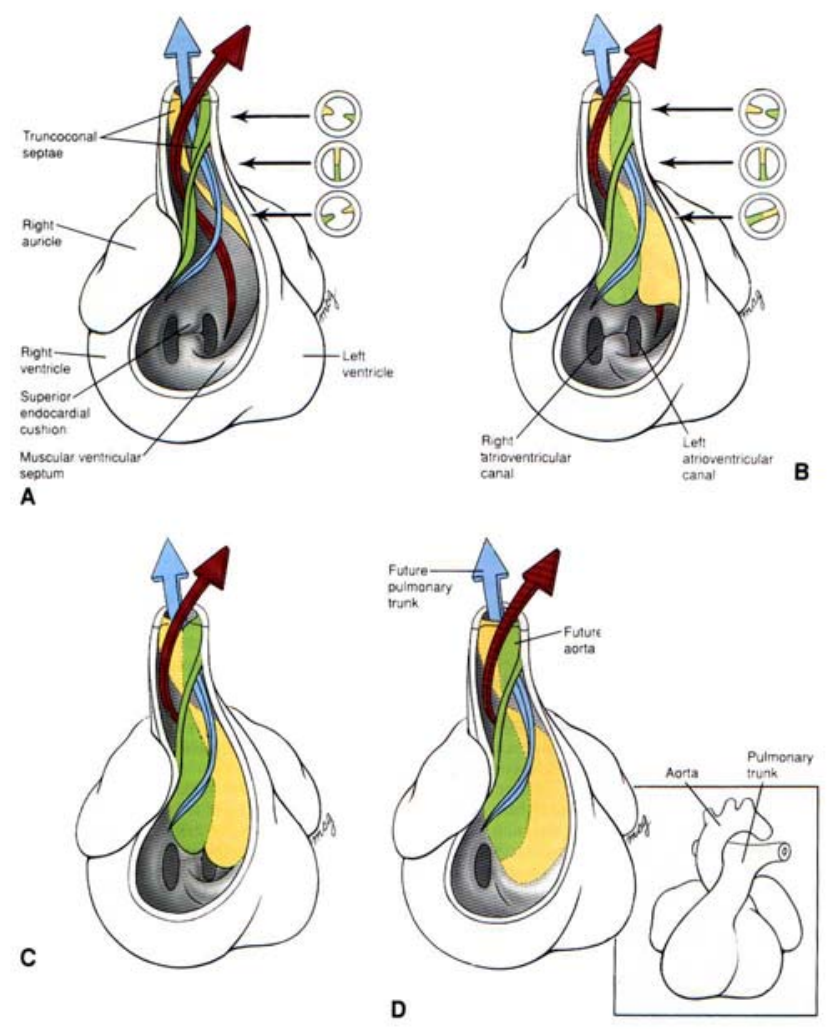

Figure 1
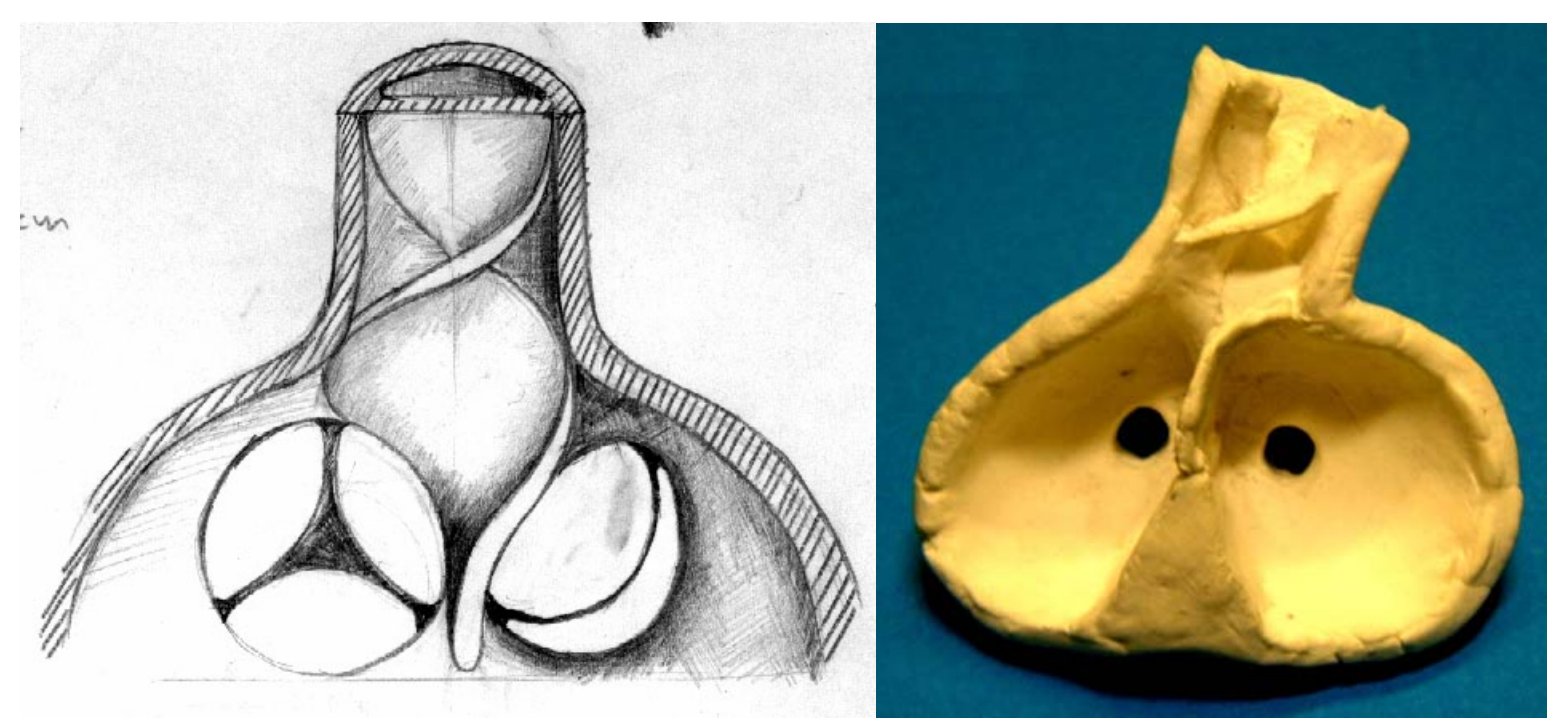

Figure 2 


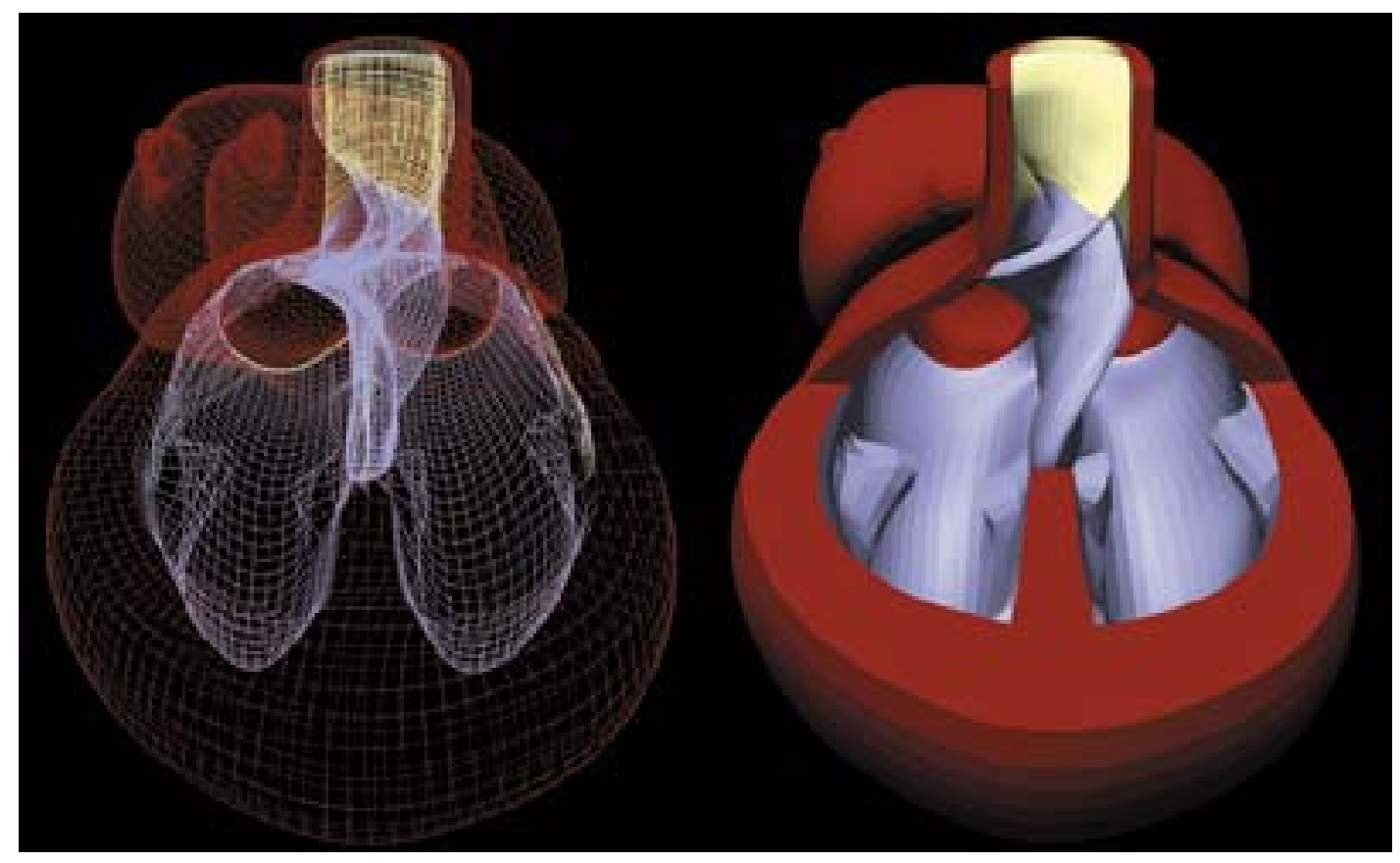

Figure 3

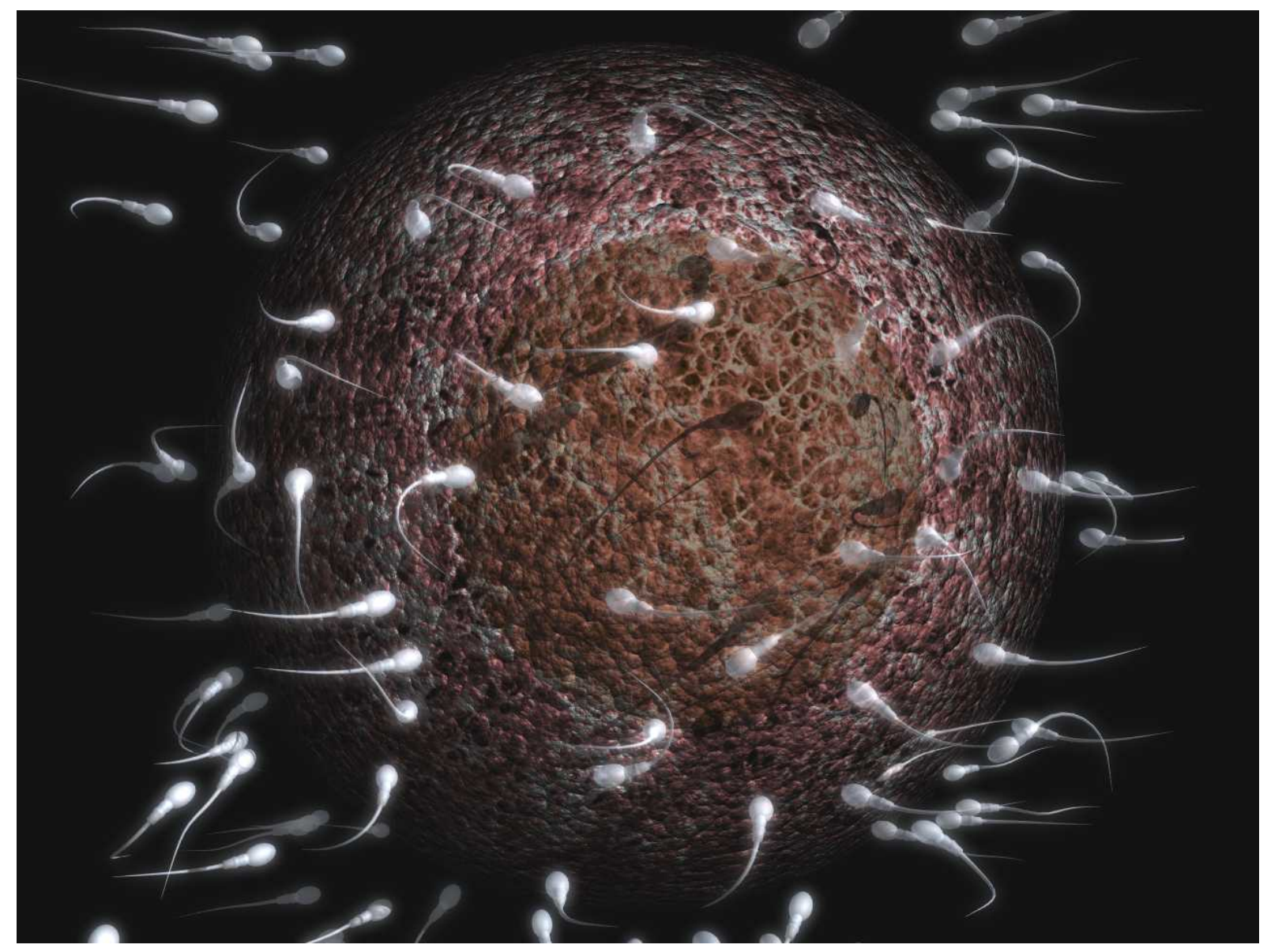

Figure 4 


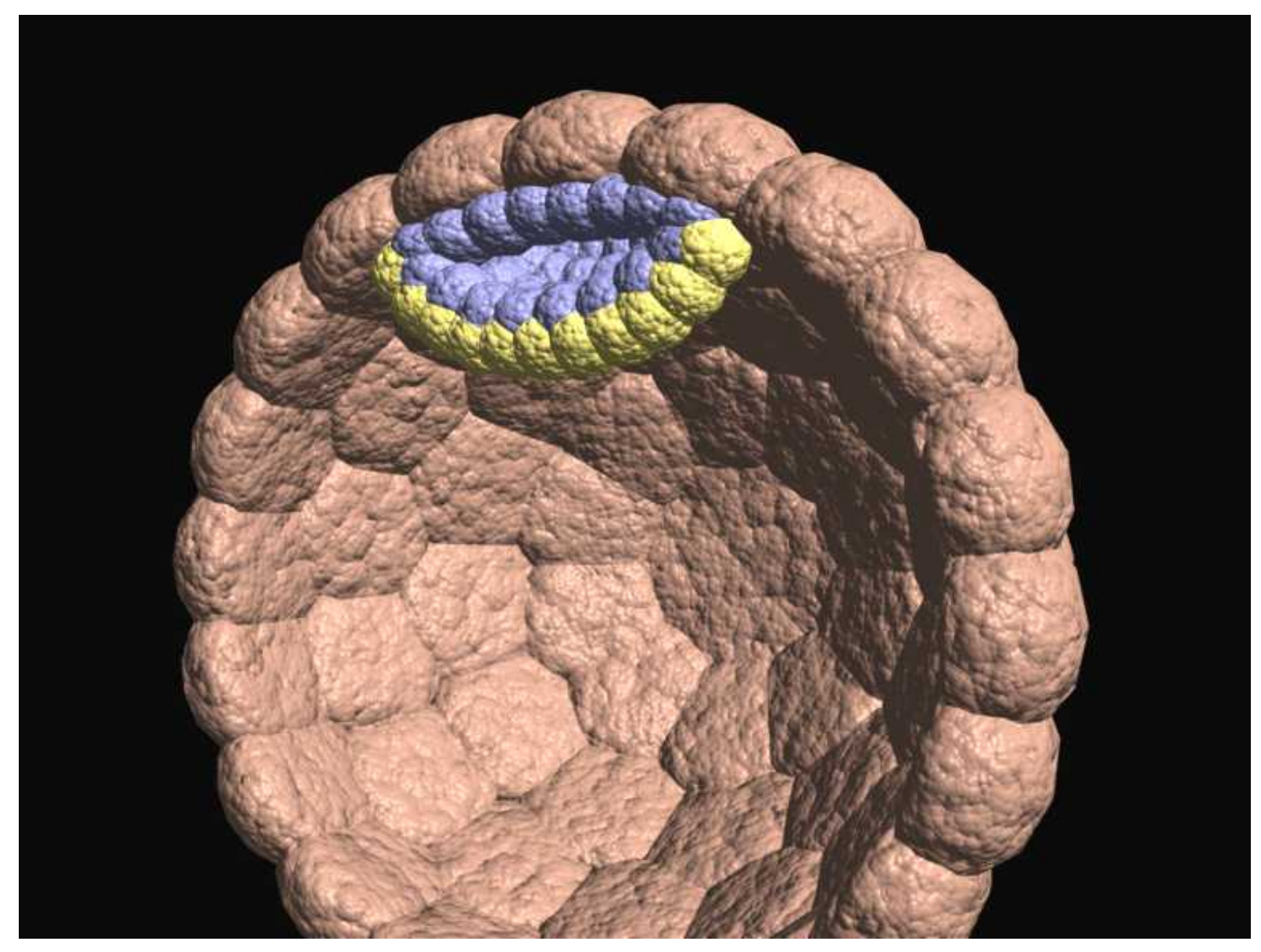

Figure 5 


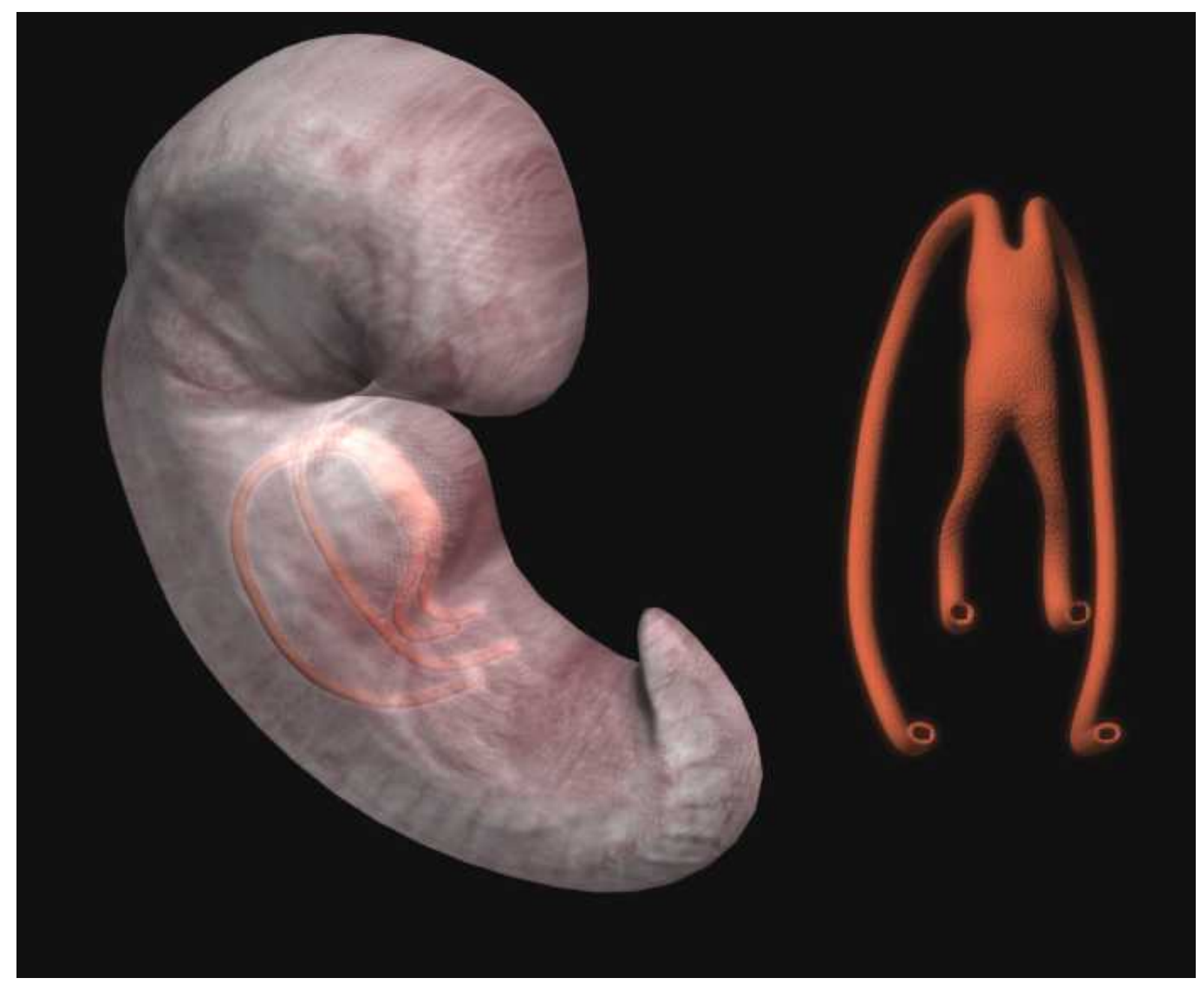

Figure 6

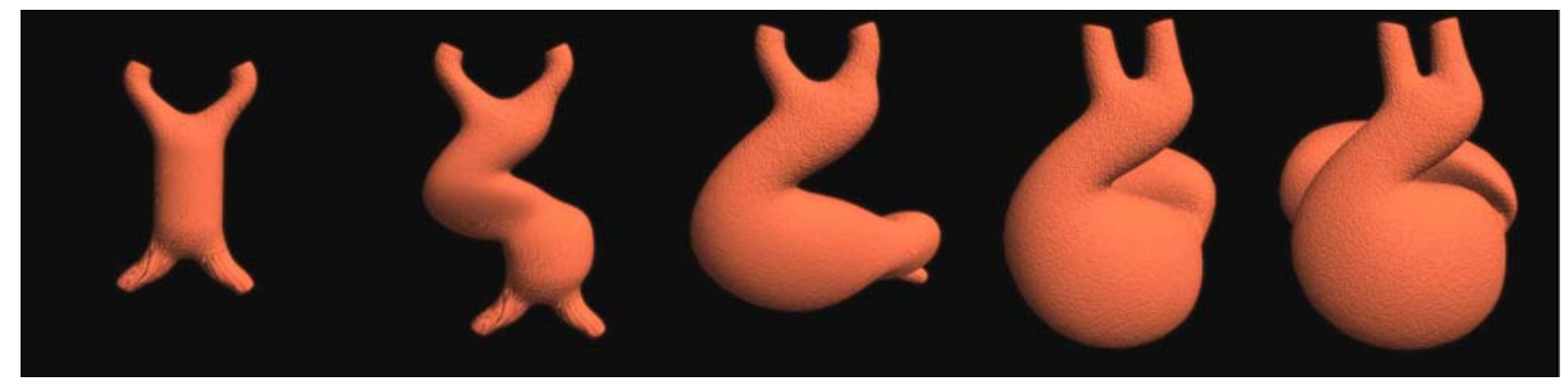

Figure 7 


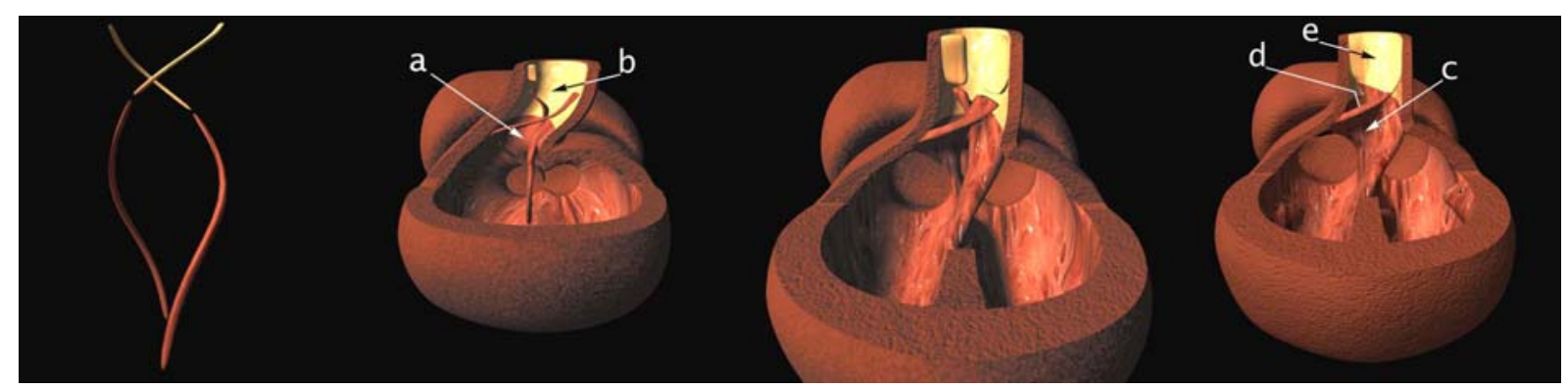

Figure 8

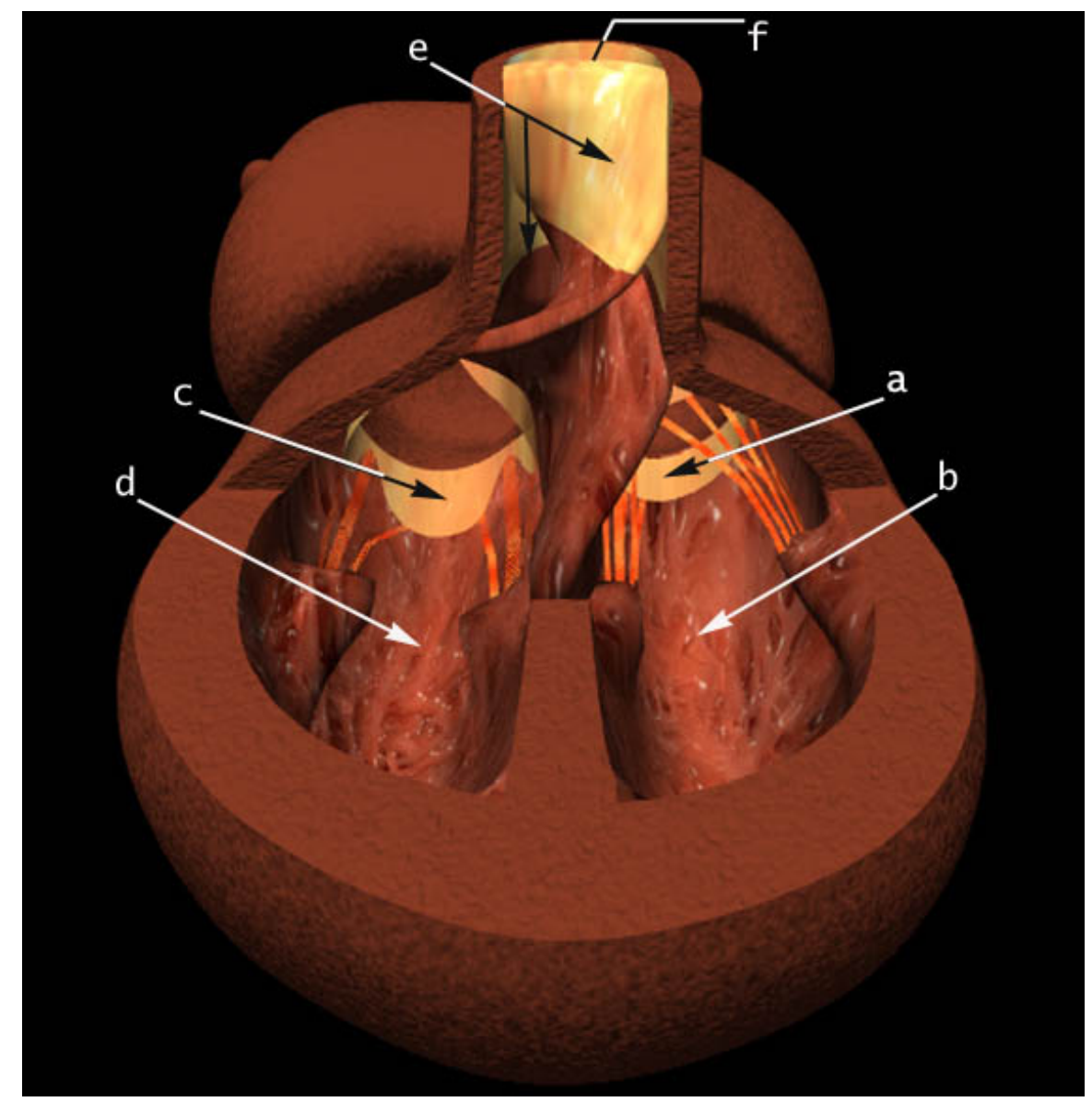

Figure 9 